\title{
State-selective effects in the differential cross section for electron capture from laser-excited sodium atoms by protons
}

\author{
R J Allan, C Courbin, P Salas and P Wahnon
}

\begin{abstract}
We investigate sodium-proton charge transfer using 14-state adiabatic, molecularbasis classical trajectory calculations in which a common translation factor and dynamical radial and rotational coupling terms are included.

The differential cross sections are evaluated by the eikonal procedure from $S$-matrix elements obtained over 283 impact parameters at each energy. A fine mesh of energy and angle resolves new effects in scattering from a target excited by a circularly-polarized laser source. One of them is ascribed to velocity matching.

Calculations were carried out on an Intel iPSC/ 2 parallel computer using a version of the EIKONXS package which is described.
\end{abstract}

Recent advances in experimental techniques have allowed the measurement of differential scattering cross sections for electron capture from laser-excited targets in well defined states. The sodium atom has featured in the first investigations due to its relative ease of excitation and large exchange cross section with protons at intermediate collision energies (Royer et al 1988, Dowek et al 1990). In a separate paper (Courbin et al 1990, hereafter referred to as I) we discuss the comparison of initial $\mathrm{Na}(3 \mathrm{~s})$ and $\mathrm{Na}(3 \mathrm{p})$ for total and differential capture cross sections into $\mathrm{H}(n=2)$ as measured in those experiments.

In the present publication new effects are presented which might be measured using similar techniques. These concern collisions in which the initial atomic orbitals can be produced using a circularly-polarized laser beam, and the final orbitals may also be detected by their emission of circularly polarized light. We show that state to state cross sections are very selective to the states involved and also to the relative collision velocity.

The amplitudes for electron capture were calculated using the classical trajectory method (I). Potential energies of the $\mathrm{NaH}^{+}$complex and dynamical coupling and translation factor terms were evaluated using a now well known model-potential technique (see references in I). The differential cross sections were obtained by the eikonal method (Piacentini and Salin 1977). Since we have used straight-line trajectories for the nuclear motion all the eikonal phases are known. All parts of the dynamical code are incorporated into a package called EIKONXs (Allan et al 1985, Allan 1990).

We have already established that the model-potential method is sufficiently accurate to describe collisions of alkali atoms and protons providing the proton does not penetrate the electronic charge cloud of the alkali. Accurate evaluation of the differential 
scattering processes therefore requires only a sufficiently detailed computation of the dynamical quantities. This in general requires a large number of states to be included to account for excitation and exchange to higher levels (we took 14 states of positive symmetry with respect to reflection in the collision plane), and also requires the amplitudes to be calculated for a large number of impact parameters so that the eikonal transformation may be made accurately (we took up to 283 impact parameters in the present work).

The calculation described above would take around $6 \mathrm{~h}$ per energy on a conventional vector computer such as the Convex $\mathrm{C} 220$. To allow more 'interactive' study of the collision mechanism we needed to reduce this time and therefore ported the program package to a parallel machine.

The Intel iPSC/2 Parallel Hypercube computer is one of a range of high-performance, low-price multicomputers which has become available over the last few years. It is built from a number of independent computing engines each with memory, floating-point CPU and hardware to route data between them, connected as vertices of a hypercube (Intel 1989). These units operate concurrently, with synchronization and exchange of data by messages (see, for instance, Allan (1989) or Allan et al (1990) for a description of some general programming techniques). Use of such a locally affordable machine allows large-scale computation and data analysis (for instance on graphics workstations) which would previously have required national resources.

Effective parallelization of our collision code was achieved by farming out different impact-parameter calculations to different processors. Since the entire code and data fits comfortably within the allowed 4 Mbyte memory per node of the Intel machine this is rather easy and yields an asymptotic linear speedup with increasing number of processors. Some overhead is seen in reading the molecular data, which is needed by all processors, as mentioned by Allan and Courbin (1989). The values of transition amplitudes for all impact parameters are stored in files on the Intel concurrent file system (Moody 1989) for use by other modules of the program.

One such is the DIFFXs module which evaluates the eikonal differential cross sections (DCs). Parallelization of this is achieved by giving each processor a different initial and final condition to work with. This is not the most efficient method, but has been adequate in the present work. Thus each processor reads the data and calculates all scattering angles. Results are stored in a file on the host computer (System Resource Manager) for later analysis.

The first part of the scalar code was found to be four times faster on 32 Intel processors as compared with a sequential version running on one processor of a Convex C220 (Allan and Courbin 1989).

The transition amplitudes and cross sections are obtained between states defined in the molecular frame and have to be projected into the fixed frame of observation. This projection is achieved using equation (4) of Wahnon et al (1988) in the case of $\mathrm{p}$ states to obtain the circularly polarized states in the collision plane, $\left|n \mathrm{p}_{-1}\right\rangle$ or $\left|n \mathrm{p}_{+1}\right\rangle$. These atomic states would be excited by a left or right circularly polarized laser beam respectively, propagating perpendicular to the collision plane in the direction of the positive laboratory-fixed quantization axis.

Probabilities of transitions with different initial and final polarization directions are shown in figure $1(a)-(d)$. The four figures are remarkably different, especially in the region of the long-range scattering ridge at $b=13.5 a_{0}$. This ridge is present in the surfaces starting from a left circularly polarized target $\left|p_{-1}\right\rangle$, but almost completely absent from those from a right circularly polarized one $\left|p_{+1}\right\rangle$, having been replaced by 
(a)
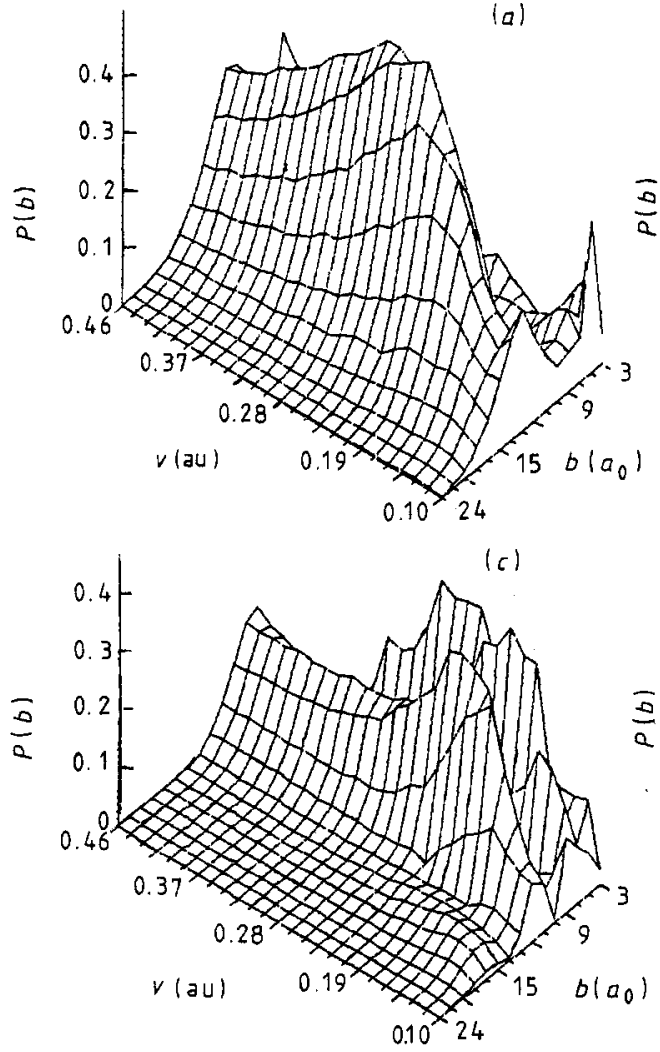

(b)

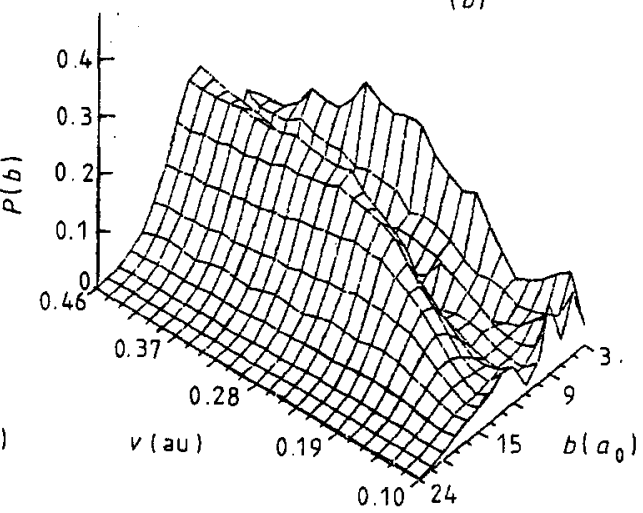

(d)

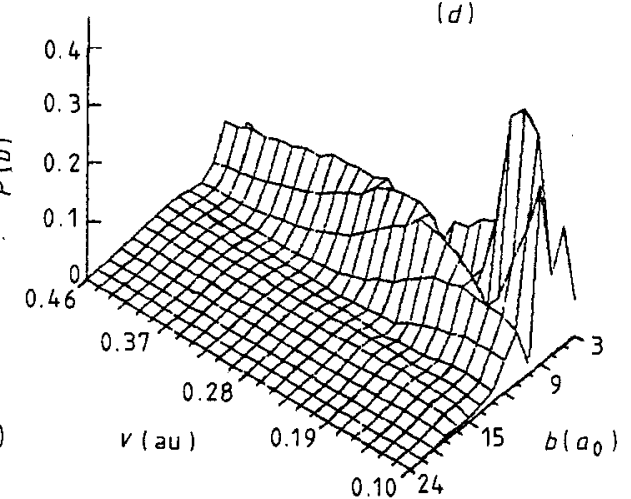

Figure 1. Probabilities for electron capture from initial circularly polarized $\mathrm{Na}(3 \mathrm{p})$ states to final circularly polarized $\mathrm{H}(2 \mathrm{p})$ states plotted as a function of collision velocity from 0.1 to 0.46 au and impact parameter from 3 to $24 a_{0} .(a)\left|p_{-1}\right\rangle$ to $\left|p_{-1}\right\rangle$ polarization in the collision plane, $\left|\mathbf{p}_{-1}\right\rangle$ corresponding to the same rotation direction as the internuclear axis (see text). (b) $\left|\mathrm{p}_{-1}\right\rangle$ to $\left|\mathrm{p}_{+1}\right\rangle,(c)\left|\mathrm{p}_{+1}\right\rangle$ to $\left|\mathrm{p}_{-1}\right\rangle,(d)\left|\mathrm{p}_{+1}\right\rangle$ to $\left|\mathrm{p}_{+1}\right\rangle$.

a dominant ridge at $b=9 a_{0}$. Not surprisingly the scattering features move towards higher impact parameter for the lower velocities as the longer interaction time strengthens the otherwise weak long-range scattering.

The most selective surface is for the $\left|n p_{-1}\right\rangle$ to $\left|n p_{-1}\right\rangle$ scattering, in which the long-range peak is most pronounced at $v=0.27$ au.

Preparing $\mathrm{Na}(3 \mathrm{p})$ with circularly polarized light, the collision plane being perpendicular to the laser beam, a classical point of view would predict that the charge exchange would take place with largest probability (for instance at $\theta>0$ with an average repulsive trajectory) when the electron in the initial orbit had an angular velocity in the same sense as the incident proton (the $\left|3 p_{-1}\right\rangle$ state in our notation) and about the same order of magnitude, compared with the other sense of rotation of the electron. The velocity of the electron in the $\left|3 \mathrm{p}_{ \pm 1}\right\rangle$ states is $v_{3 \mathrm{p}}=0.33 \mathrm{au}$, the corresponding energy of the incident proton is approximately $E=3 \mathrm{keV}$ for matching of the velocities. This compares well with the position of the peak in figure $1(a)$ which appears to be at a relative velocity of $v=0.27 \mathrm{au}$.

This matching can only happen for the case of the initial orbital angular momentum in the same sense as the nuclear axis rotation, $\left|\mathrm{p}_{-1}\right\rangle$ and is the primary mechanism by 
which the electron may remain between the nuclei long enough to be captured, therefore there is a long-range ridge in figure $1(a)$ and $(b)$ but not in $1(c)$ and $(d)$. Transfer takes place over a long distance and mixes the relative phase of the $\sigma$ and $\pi$ orbitals formed on the second centre.

A secondary post-collisional effect influences the probability of forming a final $\left|p_{-1}\right\rangle$ or $\left|p_{+1}\right\rangle$ orbital from $\left|p_{-1}\right\rangle$ at long range and has been identified by Kohmoto and Fano (1981) and Hermann and Hertel (1980) among others. The $\mathrm{H}(2 \mathrm{p})$ charge cloud will tend to preferentially rotate following the positively charged $\mathrm{Na}^{+}$ion, and therefore will tend to be also a $\left|p_{-1}\right\rangle$ state. In general, for charge transfer with velocity matching, the final orbital rotation will preferentially be in the same sense as the initial one due to this effect. We note however that there is still some $\left|\mathrm{p}_{+1}\right\rangle$ formation around $b=13.5 a_{0}$ although with lower probability.

For short-range collisions around $b=3 a_{0}$ there is an increased probability for transitions which change the sign of the polarization (figure $1(b)$ and $(c)$ ) compared with the others near to the matching velocity. Explanation of this is in terms of the molecular orbital data. In this velocity range the effect of rotational coupling is strong. Consider the coupling terms:

$$
\left\langle 3 \mathrm{p}_{-1}\left|\mathrm{i} L_{y}\right| 2 \mathrm{p}_{+1}\right\rangle=\left(\left\langle 3 \mathrm{p} \pi\left|\mathrm{i} L_{y}\right| 2 \mathrm{p} \sigma\right\rangle+\left\langle 3 \mathrm{p} \sigma\left|\mathrm{i} L_{y}\right| 2 \mathrm{p} \pi\right\rangle\right) \mathrm{i} / 2
$$

and

$$
\left\langle 3 \mathrm{p}_{-1}\left|\mathrm{i} L_{y}\right| 2 \mathrm{p}_{-1}\right\rangle=-\left(\left\langle 3 \mathrm{p} \pi\left|\mathrm{i} L_{y}\right| 2 \mathrm{p} \sigma\right\rangle-\left\langle 3 \mathrm{p} \sigma\left|\mathrm{i} L_{y}\right| 2 \mathrm{p} \pi\right\rangle\right) \mathrm{i} / 2 .
$$

Inspection of the numerical data shows that the two molecular coupling terms on the right-hand side have approximately the same magnitude and are of the same sign for small impact parameters. Thus the first combination will predominate. Similarly the $\left|3 p_{+1}\right\rangle$ to $\left|2 p_{-1}\right\rangle$ transition is more probable than the $\left|3 p_{+1}\right\rangle$ to $\left|2 p_{+1}\right\rangle$ transition. This may be fortuitous and could be different in other collision systems and for other combinations of states.

At low velocity, for the short-range collisions, it appears that the initial wavefunction retains its character but just changes its origin to the other nucleus for charge transfer. There is likely to be a small rotation of the orbitals following the internuclear axis due to the lock-in effect (e.g. Allan et al 1985) but there is no change of phase between $\sigma$ and $\pi$. Thus there is a low-velocity peak in the $\left|3 p_{-1}\right\rangle$ to $\left|2 p_{-1}\right\rangle$ and $\left|3 p_{+1}\right\rangle$ to $\left|2 p_{+1}\right\rangle$ transitions.

There is certainly no single explanation for all the features in figure 1 , and numerical calculations cannot be avoided.

Using the parallel implementation of the EIKONXs program we have been able to obtain the first detailed results of differential cross sections for initially and finally circularly polarized states. Our results substantiate the above conclusions, that is, there is a strong dependence on the sign of the initial and final circular polarization with respect to the sign of the angular momentum of the nuclei. This effect should therefore be observable with sufficient angular resolution, although there are as yet no experimental results, so a detailed discussion would be of little value.

We do however illustrate the effect of the initial condition on the electron capture to the summed $\mathrm{H}(n=2)$ states in the differential cross sections at $v=0.14$ au for the two different initial states $\left|3 p_{+1}\right\rangle$ and $\left|3 p_{-1}\right\rangle$. Figure 2 shows that the initial $\left|3 p_{-1}\right\rangle$ state leads to a much bigger cross section at angles $\theta_{\mathrm{cm}}<0.15^{\circ}$ in accordance with the increased transition probability due to velocity matching at large impact parameters. 


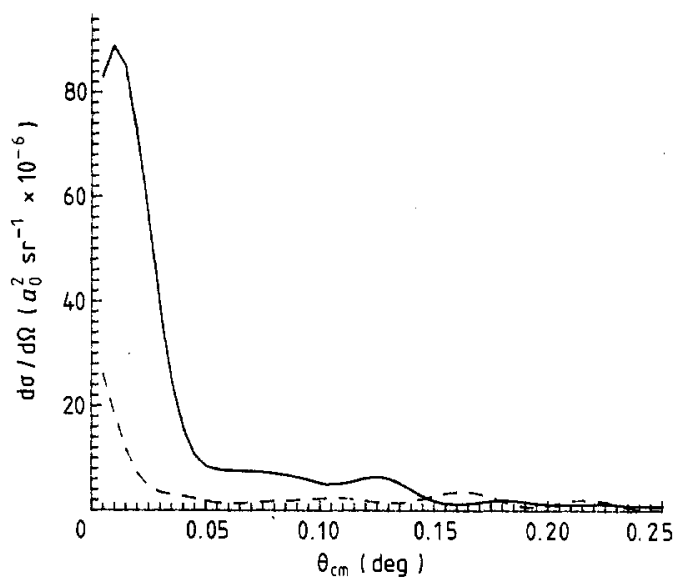

Figure 2. Differential cross sections for —_ initial $\left|3 p_{-1}\right\rangle$ state, --- initial $\left|3 p_{+1}\right\rangle$ state at $v=0.141 \mathrm{au}(E=500 \mathrm{eV})$ but now summed over final states $\mathrm{H}(n=2)$.

Experimental resolution is likely to be around $\Delta \theta_{\text {lab }}=0.1^{\circ}$ (Royer et al 1988), $\Delta \theta_{\mathrm{cm}} \approx$ $0.104^{\circ}$, for such measurements, which would emphasize this low-angle region. Confirmation of our predictions may therefore still be achieved. For the higher velocity of $v=0.27$ au closer to the full matching condition the relevant scattering angles would be four times smaller, and the effects may not currently be observable.

We have applied the molecular quasi-classical treatment to describe charge transfer from a circularly polarized laser-excited target. Results for the probability distributions and differential cross section predict some large new effects in these collisions. It is hoped that our findings will stimulate increased experimental effort to increase the understanding of the charge-transfer mechanism.

Calculations in this paper were carried out on the 64-node Intel iPSC/ 2 computer at Daresbury Laboratory. We thank the Royal Society and CNRS for financial support enabling C Courbin to visit Daresbury from September 1987 to September 1988, and the Comunidad de Madrid for supporting P Salas during his visits to Daresbury in 1987 and 1988. This work was also partially supported under EEC CODEST contract nos ST2J-0290-C(EDB) and ST2J-0033-1-UK and the Spanish DGICyT contract no PS 87-0097.

\section{References}

Allan R J 1989 FORTRAN-77 programming of parallel computers Daresbury Laboratory Technical Memorandum DL/SA/TM61T

Allan R J 1990 Documentation of the EIKONXs package Daresbury Laboratory Technical Memorandum DL/SC1/TM69T

Allan R J, Bähring A and Hanssen J 1985 J. Phys. B: At. Mol. Phys. 18 1999-2019

Allan R J and Courbin C 1989 Daresbury Laboratory Annual Report, TCS Appendix pp 70-1

Allan R J, Heck L and Zurek S 1990 Comput. Phys. Commun. 59 325-44

Courbin C, Allan R J, Salas P and Wahnon P 1990 J. Phys. B: At. Mol. Opt. Phys. submitted

Dowek D, Houver J C, Pommier J, Richter C, Royer T, Andersen N and Palsdottir B 1990 Phys. Rev. Lett. 64 1713-16

Hermann H W and Hertel I V 1980 J. Phys. B: At. Mol. Phys. 13 4285-97 
Intel Scientific Computers Ltd 1989 iPSC/2 Users Guide order number 311532-003

Kohmoto $M$ and Fano U 1981 J. Phys. B: At. Mol. Phys. 14 L447-51

Moody D 1989 Parallelogram 15 15-6

Piacentini R D and Salin A 1977 Comput. Phys. Commun. 13 57-62

Royer T, Dowek D, Houver J C, Pommier J and Andersen N 1988 Z. Phys. D 10 45-57

Wahnon P, Salas P and Courbin C 1988 Z. Phys. D 9 307-13 